\title{
SARS-CoV-2 vaccines and their challenges against the variants
}

\author{
Umid Kumar Shrestha* \\ Nepal Mediciti Hospital, Bhaisepati, Lalitpur, Nepal
}

Keywords: COVID-19; SARS-CoV-2; vaccines; variants; mutation

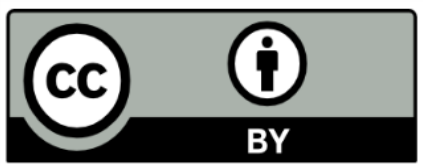

This work is licensed under a Creative Commons Attribution 4.0 Unported License.

\begin{abstract}
The coronavirus disease 2019 (COVID-19) pandemic has lead to the several researches for the development of the new severe acute respiratory syndrome corona virus 2 (SARSCoV-2) vaccines, namely mRNA vaccine, viral vector vaccine, recombinant protein vaccine and inactivated vaccine, with an objective to achieve the response which include production of neutralizing antibodies, generation of a T-cell response, and avoidance of immune-enhanced disease. Over a course of time, the SARS-CoV-2 virus has evolved and lead to mutations; the virus with one or more new mutations is referred to as a "variant" of the original virus. All new strains (P.1 from Brazil, B.1.351 from South Africa, B.1.1.7 from the UK and B.1.617 from India) have mutations in the spike protein, resulting in the threat to the effectiveness of the current available first generation vaccines. Hence, there might be the need of the development of the modified next generation of vaccines, which take care of all those variants. Nevertheless, the current first generation vaccines may still provide satisfying immunity against SARS-CoV-2 variants. Most vaccines are expected to provide protection against hospitalizations/deaths from these variants and a booster vaccine against these variants is likely to be effective. Hence, the current vaccination must proceed. As we are aware that the more the virus spreads, the more variants are likely to appear. In order to stop the spread of SARS-CoV-2 variants, it is important to get the vaccines once it is available and not to forget about the need to wash hands frequently, keep at least $1 \mathrm{~m}$ distance from others and wear a mask. With the development of the effective SARS-CoV-2 vaccines and implementation of public health measures, we can surely defeat SARS-CoV-2 virus in COVID-19 battle and end this pandemic.
\end{abstract}

The coronavirus disease 2019 (COVID-19) pandemic has lead to the several researches for the development of the new severe acute respiratory syndrome corona virus 2 (SARS-CoV-2) vaccines. During this process, spike (S) protein (particularly in its prefusion [native] conformation) was identified as the immunodominant antigen of the SARS-CoV 2 virus $^{1}$; it was found that the binding and neutralizing antibodies primarily target the receptor-binding domain (RBD) of the S1 subunit. ${ }^{2}$ After identifying the vaccine target, several SARS-CoV-2 vaccines were developed with an objective to achieve the response which include production of neutralizing antibodies, generation of a T-cell response, and avoidance of immune-enhanced disease.
The SARS-CoV-2 vaccines that have been developed to combat COVID-19 are shown in the table $1 .^{3}$

Over a course of time, the SARS-CoV-2 evolve and lead to mutations; the virus with one or more new mutations is referred to as a "variant" of the original virus. The new SARS-CoV-2 variants B.1.427 and B.1.429 (the "California" or "West Coast" variants) share S gene non-synonymous mutations at sites 13, 152, 452, and 614 and were seen during the December 2020 to February 2021 period when California was experiencing a huge peak. A modest increase in transmissibility was noted in those variants. ${ }^{4}$ The B.1.1.7 variant (also known as 501Y.V1, having a N501Y mutation in the RBD) emerged in the UK. ${ }^{5} \mathrm{~N} 501 \mathrm{Y}$ signifies that there is a change in their

\footnotetext{
${ }^{*}$ Corresponding Author:

Umid Kumar Shrestha, MD, PhD

Nepal Mediciti Hospital, Bhaisepati, Lalitpur, Nepal

E-mail: umidshrestha@gmail.com
} 
501 st amino acid from asparagine (an amide-containing amino acid) to tyrosine (a phenol-containing amino acid). This variant has eight spike mutations that include two deletions, one of which is in an antibody supersite epitope (Y144) and the other of which increases infectivity but has little impact on immune escape. ${ }^{1}$ The sole RBD mutation is N501Y, which also seems to increase binding to the host cell receptor ACE2. The epidemiological studies showed that B.1.1.7 was more transmissible. ${ }^{6}$

Table 1: SARS-Co-V-2 vaccines

\begin{tabular}{|c|c|c|}
\hline Vaccine name & Developer & Antigens \\
\hline \multicolumn{3}{|l|}{ mRNA vaccine: } \\
\hline BNT162b1 & BioNTech/Pfizer & RBD trimer \\
\hline BNT162b2 & BioNTech/Pfizer & S-full length (pre-fusion) \\
\hline mRNA-1273 & Moderna & S-full length (pre-fusion) \\
\hline \multicolumn{3}{|l|}{ Viral vector vaccine: } \\
\hline Ad5-nCoV & CanSino & S-full length \\
\hline Ad26.COV2.S & Janssen/Johnson \& Johnson & S-full length (pre-fusion) \\
\hline AZD-1222 / ChAdOx1-S & $\begin{array}{l}\text { Oxford/AstraZeneca } \\
\text { (brand name COVISHIELD) } \\
\text { is manufactured by } \\
\text { Serum Institute of India in } \\
\text { collaboration with Oxford/ } \\
\text { AstraZeneca) }\end{array}$ & S-full length \\
\hline Sputnik-V & Gamaleya & S-full length \\
\hline \multicolumn{3}{|l|}{$\begin{array}{l}\text { Recombinant protein } \\
\text { vaccine: }\end{array}$} \\
\hline NVX-CoV2373 & Novavax & S-full length (pre-fusion) \\
\hline \multicolumn{3}{|l|}{ Inactivated vaccine: } \\
\hline $\begin{array}{l}\text { Inactivated vaccine (Vero } \\
\text { cell) }\end{array}$ & $\begin{array}{l}\text { Wuhan Institute of Biological } \\
\text { Products (WIBP) }\end{array}$ & $\begin{array}{l}\text { Inactivated SARS-CoV2 } \\
\text { protein }\end{array}$ \\
\hline BIBP-CorV (Vero cell) & $\begin{array}{l}\text { Beijing Institute of Biological } \\
\text { Products (BIBP) }\end{array}$ & $\begin{array}{l}\text { Inactivated SARS-CoV2 } \\
\text { protein }\end{array}$ \\
\hline CoronaVac (Vero cell) & Sinovac & $\begin{array}{l}\text { Inactivated SARS-CoV2 } \\
\text { protein }\end{array}$ \\
\hline $\begin{array}{l}\text { Inactivated vaccine (Vero } \\
\text { cell) }\end{array}$ & $\begin{array}{l}\text { Institute of Medical Biology, } \\
\text { Chinese Academy of Medical } \\
\text { Sciences (IMBCAMS) }\end{array}$ & $\begin{array}{l}\text { Inactivated SARS-CoV2 } \\
\text { protein }\end{array}$ \\
\hline
\end{tabular}

The two variants, B.1.351 and P.1 variants (also known as 501Y.V2 and 501Y.V3) emerged in South Africa and Brazil having additional mutations in the RBD at positions E484 and K417, respectively. Viral variants with the triple combination of N501Y, E484K and K417N/T have significantly reduced susceptibility to vaccineinduced and convalescent sera. ${ }^{7}$ Other variants of concern are P.3 in the Philippines, B.1.526 in the USA and B.1.525 in the UK and west Africa, having mutations of N501Y and E484K. The new variant, called B.1.617, was initially detected in India with two mutations - the E484Q and L452R. In addition to these RBD mutations, a neglected area of SARS-CoV-2 evolution pertains to the amino-terminal domain (NTD) mutations in spike protein, which lead to the reduced sensitivity to neutralizing antibodies.

The AstraZeneca ChAdOx1 vaccine showed only $10 \%$ protection against mild-to-moderate disease associated with the B.1.351 variant in a young population with median age of 30 in South Africa. ${ }^{8}$ By contrast, in the UK, ChAdOx1 demonstrated $75 \%$ protection against B.1.1.7 (including asymptomatic infection).
The Novavax vaccine, which consists of purified spike protein, showed approximately $50 \%$ protection against infection in South Africa (largely the B.1.351 variant) and $86 \%$ protection against infection in the UK (predominantly the B.1.1.7 variant). ${ }^{9}$ Johnson \& Johnson's human adenovirus-vectored vaccine showed $64 \%$ protection against moderate-to-severe disease in South Africa (dominated by the B.1.351 variant) and $66 \%$ protection against moderate-to-severe disease in the USA (mainly the Wuhan-1 variant with $\mathrm{D} 614 \mathrm{G}$ ), as assessed 29 days after vaccination. ${ }^{10}$ The Pfizer/BioNTech BNT162b2 mRNA vaccine was reported to be less effective against B.1.351 than against non-B.1.351 variants based on a small analysis of breakthrough infections that were enriched for B.1.351 in Israel. ${ }^{11}$ The efficacy of CoronaVac/Sinovac inactivated virus vaccine in Brazil, where $75 \%$ of infections were with the P. 1 variant, was estimated at around $50 \%$ against symptomatic infection. ${ }^{12}$

The SARS-CoV-2 S protein has been the target of most firstgeneration vaccines, almost exclusively using the D614 sequence, an early variant with an aspartic acid (D) to glycine (G) mutation at position 614, D614G. The recent fast-spreading variants-including the B.1.1.7 (501Y.V1) lineage from the UK, the B.1.351 (501Y.V2) lineage from South Africa, and the B.1.1.28 (484K.V2; P.1) lineage from Brazil all contain the D614G substitution. The enhanced infectivity of the G614 virus largely results from the increased stability of the $S$ trimer, rather than the better-exposed RBDs. ${ }^{13}$ As the $\mathrm{G} 614$ strain has become dominant, the current first generation vaccines are constrained and may not target the prefusion form of the spike protein. Hence, next-generation vaccines including G614 strain would need to be designed to overcome such variants.

The mutations that would need to be included in a modified next generation of vaccines are E484K, N501Y and L452R mutations in the RBD ( $L 452 \mathrm{R}$ being found in the recently reported B.1.617 variant emerging in India and in the B.1.429 variant that has emerged in the USA), and $\mathrm{P} 681 \mathrm{H} / \mathrm{R}$ mutation in the furin cleavage site (which is found in the B.1.1.7 variant) as well as NTD deletions. ${ }^{14}$ Regularly updated consensus document from the World Health Organization should be published about the mutations to be included in the next generation of vaccines.

Despite the challenges imposed by the new variants, the current first generation vaccines may still provide satisfying immunity against SARS-CoV-2 variants. Most vaccines are expected to provide protection against hospitalizations/deaths from these variants and a booster vaccine against these variants is likely to be effective.

Hence, the vaccination must proceed. As we are aware that the more the virus spreads, the more variants are likely to appear. In order to stop the spread of SARS-CoV-2 variants, it is important to get the vaccines once it is available and not to forget about the need to wash hands frequently, keep at least $1 \mathrm{~m}$ distance from others and wear a mask. With the development of the effective SARS-CoV-2 vaccines and implementation of public health measures, we can surely defeat SARS-CoV-2 in COVID-19 battle and end this pandemic.

May 2, 2021

\section{References}

1. Du L, He Y, Zhou Y, et al. The spike protein of SARS-CoV-a target for vaccine and therapeutic development. Nat Rev Microbiol. 2009;7(3):226-236.

2. Premkumar L, Segovia-Chumbez B, Jadi R, et al. The 
receptor binding domain of the viral spike protein is an immunodominant and highly specific target of antibodies in SARS-CoV-2 patients. Sci Immunol. 2020;5(48):eabc8413

3. Li DD, Li QH. SARS-CoV-2: vaccines in the pandemic era. Military Medical Research 2021; 8:1 https://doi.org/10.1186/s40779020-00296-y

4 .Peng J, Liu J, Mann SA, et al. Estimation of secondary household attack rates for emergent spike L452R SARS-CoV-2 variants detected by genomic surveillance at a community-based testing site in San Francisco. Clinical Infectious Diseases 2021 ciab283. https://doi.org/10.1093/cid/ciab283

5. Kemp SA, Meng B, Ferriera IA, et al. Recurrent emergence and transmission of a SARS-CoV-2 spike deletion H69/V70. Preprint at bioRxiv 2021. https://doi.org/10.1101/2020.12.14.422555

6. Volz E, Mishra S., Chand M, et al. Assessing transmissibility of SARS-CoV-2 lineage B.1.1.7 in England. Nature 2021. https:// doi.org/10.1038/s41586-021-03470-x

7. Cele S, Gazy I, Jackson L, et al. Escape of SARS-CoV-2 501Y.V2 from neutralization by convalescent plasma. Nature 2021. https://doi.org/10.1038/s41586-021-03471-w

8. Madhi SA, Baillie V, Cutland CL, et al. Efficacy of the ChAdOx1 nCoV-19 Covid-19 vaccine against the B.1.351 variant. N. Engl. J. Med 2021. https://doi.org/10.1056/NEJMoa2102214.

9. Shinde V, Bhikha $S$, Hoosain $Z$ et al. Preliminary efficacy of the NVX-CoV2373 Covid-19 vaccine against the B.1.351 variant. Preprint at medRxiv 2021. https://doi.org/10.1101/2021.02.2 5.21252477.

10. Sadoff J, Gray G, Vandebosch A, et al. Safety and efficacy of single-dose Ad26.COV2.S vaccine against Covid-19. N. Engl. J. Med 2021. https://doi.org/10.1056/NEJMoa2101544.

11 .Kustin T, Harel N, Finkel $U$, et al. Evidence for increased breakthrough rates of SARS-CoV-2 variants of concern in BNT162b2 mRNA vaccinated individuals. Preprint at medRxiv 2021. https://doi.org/10.1101/2021.04.06.21254882.

12. Hitchings MDT, Ranzani OT, Torres MSS, et al. Effectiveness of CoronaVac in the setting of high SARS-CoV-2 P.1 variant transmission in Brazil: a test-negative case-control study. Preprint at medRxiv 2021. https://doi.org/10.1101/2021.04.0 7.21255081 .

13. Zhang J, Cai Yongfei, Xiao T, et al. Structural impact on SARSCoV-2 spike protein by D614G substitution. Science 2021:372 (6541); 525-530. DOI: 10.1126/science.abf2303

14. Gupta, R.K. Will SARS-CoV-2 variants of concern affect the promise of vaccines?. Nat Rev Immunol (2021). https:// doi.org/10.1038/s41577-021-00556-511. Le Bert N, Tan AT, Kunasegaran $\mathrm{K}$, et al. SARS-CoV-2-specific T cell immunity in cases of COVID-19 and SARS, and uninfected controls. Nature 2020; 584, 457-462. doi:10.1038/s41586-020-2550-z

12. Braun J, Loyal L, Frentsch $M$ et al. SARS-CoV-2-reactive T cells in healthy donors and patients with COVID-19. Nature 2020. Published online July 29, 2020. doi: 10.1038/s41586-0202598-9.

13. Bastard P, Rosen LB, Zhang Q. Auto-antibodies against type I IFNs in patients with life-threatening COVID-19. Science 2020. Published online on September 24, 2020; eabd4585. doi: 10.1126/science.abd4585.

14. Zhang Q, Bastard P, Liu Z, et al. Inborn errors of type I IFN 